\title{
A computational model of cooperative spatial behaviour for virtual humans
}

\author{
Nhung Nguyen and Ipke Wachsmuth
}

\begin{abstract}
This chapter introduces a model which connects representations of the space surrounding a virtual humanoid's body with the space it shares with several interaction partners. This work intends to support virtual humans (or humanoid robots) in near space interaction and is inspired by studies from cognitive neurosciences on the one hand and social interaction studies on the other hand. We present our work on learning the body structure of an articulated virtual human by using data from virtual touch and proprioception sensors. The results are utilized for a representation of its reaching space, the so-called peripersonal space, a concept known from cognitive neuroscience. In interpersonal interaction involving several partners, their peripersonal spaces may overlap and establish a shared reaching space. We define it as their interaction space, where cooperation takes place and where actions to claim or release spatial areas have to be adapted, to avoid obstructions of the other's movements. Our model of interaction space is developed as an extension of Kendon's F-formation system, a foundational theory of how humans orient themselves in space when communicating. Thus, interaction space allows for measuring not only the spatial arrangement (i.e., body posture and orientation) between multiple interaction partners, but also the extent of space they share. Peripersonal and interaction space are modeled as potential fields to control the virtual human's behaviour strategy. As an example we show how the virtual human can relocate object positions toward or away from locations reachable for all partners, and thus facilitating cooperation in an interaction task.
\end{abstract}


Acting smoothly in space and avoiding collisions with others in their workspace (the space where movements are carried out) is a crucial aspect of natural spatial behaviour of any articulated agent. Just think of two interactants building a toy tower together, where they are able to coordinate parallel actions in shared space without getting in the way of each other. Humans accomplish such tasks without explicit negotiation. For instance, they place needed component parts to areas where the other can reach them or see them and at the same time avoid obstructing each other's workspace or view. Such spatial behaviour in shared space interaction can fulfil two functions: first, the function of coordinating the interactants' actions to successfully accomplish a joint task; second, the function of conveying social signals.

In this chapter we shall demonstrate how a virtual human can cooperate with a partner in building a toy tower together, as one aspect of computationally modelling shared space and spatial behaviour for action coordination. Virtual humans are autonomous agents with human-like appearance and usually human-like multi-modal behaviour like speech, gaze, gestures, and facial expressions. In three-dimensional virtual reality environments, virtual humans can interact with other virtual humans or with real humans. For example, virtual humans like Max (Kopp et al., 2003) can act as co-situated guides in a construction task, or Steve (Rickel and Johnson, 2000), who act as tutors demonstrating physical tasks to students.

In the mentioned scenarios, overlapping workspaces were usually avoided by maintaining enough distance between the partners to avoid interferences between their movements. We believe that in natural interaction such interferences have to be dealt with to accomplish cooperative interaction tasks. Thus, we present our work on modelling a virtual human's spatial behaviour in shared near space interactions in order to facilitate the accomplishment of and the partner's engagement in the cooperative task.

Spatial interaction in tasks carried out in distances near to the agent's body usually pose a great challenge to virtual humans. In contrast, humans seem to manage these tasks without much effort. As Tenbrink, Wiener, and Claramunt state in the Introduction to this volume, computational models rely strongly on empirical findings of spatial cognition such as those presented in Part 1 'Empirical Insights'. Such findings 
serve as helpful evidence for building human-like behaviour for virtual humans. Our approach specifically takes inspiration from findings on how humans process spatial interaction tasks on the level of spatial perception and representation, which again has consequences on the design of virtual humans' spatial perception and representation. Since the findings originate from various disciplines, such as neuropsychology, cognitive neuroscience, and cognitive science, one typical issue for computer scientists is to translate the concepts into technical models. Two main aspects related to human spatial cognition are technically modelled in the present work. The first aspect is concerned with how the reach-space (peripersonal space) is represented and structured in humans (see Section 4.3.1). The second aspect deals with how humans represent the peripersonal space for a proximate partner when interacting with her. This matter is addressed under the concept of spatial perspective taking in Section 5.1.

Although our computational model integrates theoretical concepts from nontechnical disciplines, one main goal for technical systems eventually is the application in interaction with a human partner. One common view, especially in the field of virtual human development, is to build agents that are able to assist and guide humans. This view is also reflected in Part 3 'Intuitive Assistance'. Following the definition of Bhatt, Schultz, and Freksa (this volume) our virtual human application could therefore be conceived of as a Spatial Assistance System, within the domain of Ambient Assisted Living (AAL). According to Bhatt and colleagues, AAL applications usually involve software systems or robots which are supposed to assist and empower humans within private spaces in everyday life. We adopt this definition and in addition believe that in AAL applications especially with embodied agents, the engagement of humans increases since humanoid robots and virtual humans imply more immediate spatial interaction. In our application, Max builds a hypothesis from the partner's spatial perspective and matches it with the next actions of the joint task. If a needed object is not reachable for the partner, Max places the object to locations that are reachable for her. We believe that embodied agents that are supposed to share the same space with humans will need to deal with the spatial perspective of the partner as well as with social aspects. In some cases, especially when it comes to the private spaces mentioned by Bhatt et al., these factors might turn an assistive agent into a more cooperative and thus more acceptable partner.

Improving virtual humans in movements carried out in their individual 
workspace is a classic problem (Huang et al., 2003; Zhao et al., 2005). We put the focus of this chapter on two issues of this challenge. One issue is to improve the virtual human's sensorimotor and perceptual abilities, which are crucial for body action/ motion planning and control. The virtual human's workspace is the space where sensor modalities have to focus on and where possible objects have to be observed or manipulated by reaching, grasping, or avoiding them. Sharing parts of this space with other agents makes interaction only more challenging, which leads to the following second issue. As mentioned before, the partners' actions and movements have to be coordinated, thus, the virtual human needs a representation of the shared near space in order to perform smooth, effective, and also cooperative interaction. We focus on delimited near space arrangements (e.g. a table), involving mainly the virtual human's upper part of the body.

In our work we connect the two issues of modelling the space surrounding the body with regard to an individual virtual human and modelling the same space with regard to interpersonal interaction. Accordingly, our goals in developing a virtual human are first, to enable the virtual human to learn and adapt to its reaching space, that is, the virtual human knows from its sensor modalities whether objects are in the near space immediately surrounding its body or whether it is beyond this space, second, to facilitate its perception and action within its reaching space, that is, to control the virtual human's perceptual and sensory attention with taking account of possible objects and hindrances, and third, to facilitate cooperation in shared space, for example by sustaining actions toward locations reachable by all interaction partners.

In the presented approach we approve the recent work outlined by Lloyd (2009) claiming that the principles underlying the individual representation of the space surrounding the human body also mediate the space between interacting human partners. This idea is also valuable to provide virtual humans with the abilities we aim to model. We present how our work on the reaching space of an individual articulated agent's body - the peripersonal space - is used to model the shared reaching space of cooperative interaction partners, which we define as interaction space.

Motivated by research from biology, neuro-, and cognitive science our model of peripersonal space is in particular derived from the cognitive model of egocentric space by Previc (1998) and takes input from the virtual human's sensor modalities to learn its reaching and lean-forward distances. One key feature of our computational model is the 
partition of peripersonal space into different subspaces. Although peripersonal space concepts have been studied in different research fields, they are widely unexplored in situations of face-to-face interaction. With regard to this aspect our work on interaction space is developed as a supplement to Kendon's F-formation system, a concept describing and analysing spatial arrangements in human interaction (Kendon, 1990). The F-formation system describes how humans arrange their body orientation and position to each other when cooperating in physical space. In our approach, we use potential field functions to facilitate the virtual human's behaviour strategies in peripersonal and interaction space. Depending on its own interaction goals, layout and position of the interaction space, the virtual human can plan its actions, for example, relocating object positions toward or away from locations reachable for all partners. This application demonstrates how the virtual human facilitates cooperative interaction in a joint task with other partners.

The remaining chapter is organized as follows. In the next section we briefly explain the terms and concepts from other research disciplines on which we base our presented work and we describe related work in modelling artificial humanoids. In Section 3 we propose an interpretation of the concepts, suitable for a technical framework. In Section 4 the work on how a virtual human learns its peripersonal space is presented. Based on the learned reaching distances, we show how information from multiple sensor modalities is organized in spatial maps to help maintain the virtual human's attentional focus and perception in peripersonal space. In Section 5 we present our novel approach on a computational model of interaction space by supplementing Kendon's F-Formation system using potential fields. Finally, in Section 6 we summarize the major aspects of our approach.

\section{From body space to interpersonal space}

In this section we highlight relevant definitions and valuable findings from technical as well as non-technical research areas on the space immediately surrounding a body. In the following we use the term body space when generally refering to this space, to avoid misunderstandings. It can be observed that individual body space is often analysed in terms of sensor-motor and perceptual characteristics, and commonly termed as peripersonal space, for example in engineering, cognitive neurosciences, or biology. In 
contrast, when body space co-occurs in interaction with others, it is usually analysed as a social phenomenon and treated in terms of social relationships depending on body distances and orientations. Of particular interest are recent works that highlight the so far unattended influence of shared body spaces on social cognitive processes.

\section{$2.1 \quad$ Body schema and peripersonal space}

Holmes and Spence (2004) presented evidence for a neural multi-sensory representation of peripersonal space that codes objects in body-centred reference frames and defines humans' actions in near space: 'Objects within peripersonal space can be grasped and manipulated; objects located beyond this space (in what is often termed 'extrapersonal space') cannot normally be reached without moving toward them' (Holmes and Spence, 2004: 94). As stated by Biocca et al. (2005) findings from research in human spatial cognition suggest that objects are represented using different types of spatial reference systems. For example, according to Longo and Lourenco (2007) peripersonal space is represented differently from extrapersonal spaces which also supports prior work by Previc (1998). Vallar and Maravita (2009) propose that the spatial environment proximate to humans is represented in egocentric (i.e. body-centred) frames of reference, whereas distances where spatial navigation is carried out are represented in allocentric (i.e. independent from observer) frames of reference.

Previc introduced a comprehensive theoretical model of humans' threedimensional spatial interactions containing four different realms. His model is a synthesis of existing models and findings from cognitive neuroscience and neuroscience. In addition to peripersonal (PrP) space he distinguishes three extrapersonal spaces differing in function and extent (i.e. size). Of particular interest is that he defines PrP's lateral extent as being $60^{\circ}$ central in front of the body, corresponding to the extent of human stereoscopic vision. PrP, together with one of the extrapersonal spaces, also includes movements of the upper torso, for example leaning forward to reach for objects, which Holmes and Spence assign to extrapersonal space.

One foundation of Previc's interaction-focused model comprises findings about sensor-modality specific space representations. More recent findings in this vein are presented by Avraamides, Mello, and Greenauer (this volume). In particular the reviewed literature on the functional equivalence between different sensor modalities in 
building a unified spatial representation, are consistent with the multi-sensory representation of peripersonal space. One aspect discussed by Avraamides et al. is the functional equivalence between sensory and linguistic space representations which we also address in this chapter.

Work on utilizing the concept of peripersonal space as a way towards naturally structuring visual object recognition tasks in artificial systems has been conducted by Goerick et al. (2005). We use peripersonal space to structure the space covered by multiple sensor modalities.

Although research on the different representations and functions of peripersonal and extrapersonal spaces allow deeper insights, the boundaries between them is still a matter of discussion. For example, work from Caggiano et al. (2009) suggest that peripersonal space's boundary is limited to the arms' reach. In contrast, Longo and Lourenco (2006) did not discover a categorical limit but instead found that peripersonal space is scaled as a ratio of arm's length and gradually transitions to extrapersonal spaces. Their work raises a less rigid definition of peripersonal space which changes with movements of the torso and tool use. In humans, the representation of peripersonal space is intimately connected to the representation of the body structure, namely the body schema. A comprehensive discussion on body schema, as a neural representation, which integrates sensor modalities, such as touch, vision, and proprioception, was provided by Gallagher (2005). This integration or mapping across the different modalities is adaptive to changes of the body, that is, if the structure of the body changes, the representation also changes. Findings from Maravita and Iriki (2004) revealed the adaptivity of body schema in tool use. They showed that the body schema representation extends and incorporates a tool wielded by monkeys. This is in line with findings by Longo and Lourenco (2007) stating that when wielding a tool with the hand, the tool is integrated into the body schema which can be interpreted as a manipulation of the arm length and therefore extends the size of peripersonal space. A lot of research in robotics was inspired by the concept of an adaptive body schema which offers a mechanism to learn tool use and to save engineers from laborious work on predefining an artificial articulated agent's - possibly changing body structure (Nabeshima et al., 2006; Fuke et al., 2007). More recently, work with different approaches on connecting body schema learning with interpretations of peripersonal space for articulated agents have also been presented (Hersch et al., 2008; Fuke et al., 2009). Our current research 
addresses this aspect (Nguyen and Wachsmuth, 2009), which is reconsidered in more detail in this chapter.

\subsection{Interpersonal space}

In this section we introduce how body space is defined when occurring in interpersonal interaction. A prominent model on interpersonal space is Hall's model of proxemics (Hall, 1966), which describes interpersonal distances starting from what he calls an intimate distance of a few inches to large-scale distances from 25 feet $(7.6 \mathrm{~m})$ and more. The range of peripersonal space falls roughly into the scope of intimate and personal distance. Hall's theory is a taxonomy which maps interpersonal distances to human social relationships. Therefore, it does not aim at analysing the cognitive relevance of the spaces. An example of robots changing their locomotion in the presence of humans, depending on social spaces, has been presented by Sisbot et al. (2006). We will not focus on locomotion or navigation, but instead we focus on face-to-face interaction in which a virtual human changes its motor actions depending on the space it shares with others.

Aware of the two isolated fields of neural analysis of peripersonal space and research on interpersonal behaviour, Lloyd proposes a framework that aims to investigate and interpret the 'neural mechanisms of 'social space' '(Lloyd, 2009: 298). In her hypothesis she argues that the mechanism explaining how interactions with inanimate objects affect body space, can be applied to interactions with human partners. Krueger (2011: 2) addresses the same issue by stating: 'Yet little attention has been paid to what sort of role these shared action-spaces themselves might play in driving various social cognitive processes'. This issue is a major aspect in our framework.

Kendon (1990) presented a notably relevant work on observable patterns, called formations, when humans orient and group themselves in physical space. He defines an F-formation as a pattern, which 'arises whenever two or more people sustain a spatial and orientational relationship in which the space between them is one to which they have equal, direct, and exclusive access' (Kendon, 1990: 209). He describes in particular three typical F-formations, namely vis-à-vis, L-, and side-by-side arrangements, as depicted in Figure 1. Kendon also mentions an activity space in front of a single interactant, which he calls a transactional segment. This space somehow 
corresponds to peripersonal space, as previously defined. In arrangements, where several interactants' transactional segments overlap, the intersection is called o-space (see the grey regions in Figure 1). Kendon mentions, but does not elaborate on the two spaces. We amend these aspects by focusing on the space between F-formations in Sections 4.3 and 5.

Other work has been presented, using Kendon's F-formation system for proximity control of agents, which navigate in space in the presence of humans or other agents. In the work of Yamaoka et al. (2008) a robot engages the most appropriate position and orientation to present products to customers or exhibits to museum visitors. Virtual humans have also been provided with navigation models which incorporate Fformations. Pedica and Vilhjálmsson (2009) aim at modelling groups of virtual characters that show a certain degree of social presence in virtual reality environments like games. To achieve their goal they presented a behaviour model which controls group formations of virtual characters. In contrast to these works, we do not deal with creating an F-formation, but with extending o-space and sustaining cooperative interaction, once an F-formation is established.
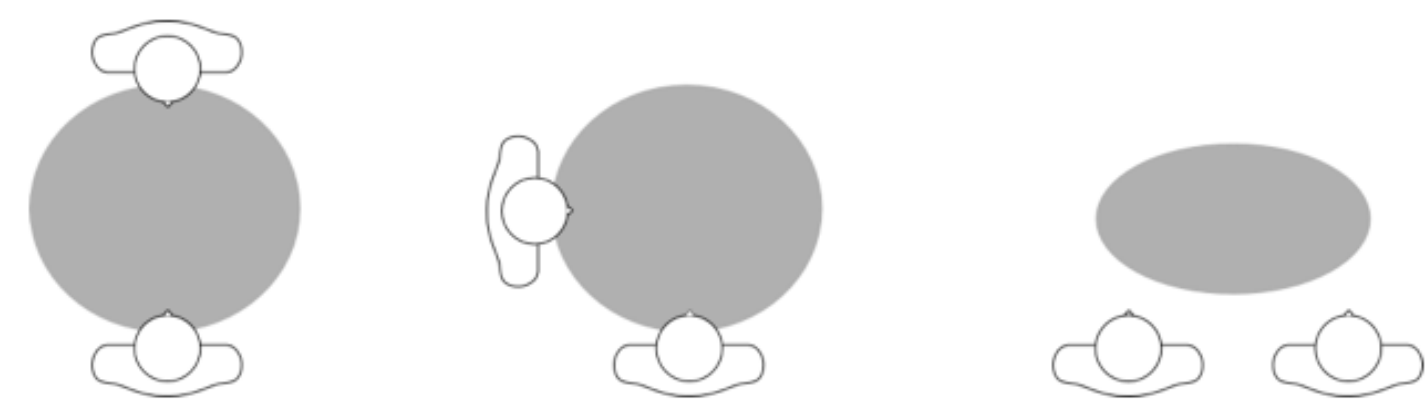

Figure 1: Spatial arrangements typical in F-Formations. From left to right: A vis-à-vis, L- and side-by-side arrangement.

\section{Technical framework}

We first present an overview of the architecture to realize a technical system which models peripersonal space and interpersonal space at the same time (see Figure 2). In the following subsections we will describe the different parts in more detail. The findings from other research fields, presented in the previous section, are incorporated into our framework. 


\subsection{Body schema}

The virtual human learns its body structure and the kinematic functions of the limbs by means of a recalibration approach involving tactile and proprioceptive sensor data. Thus, the limb lengths and joint positions of the kinematic skeleton are learned. This part is described in Section 4 and corresponds to the findings in humans, stating that body schema is learned from sensor-motor information, coding the body's kinematic structure and is adaptive to bodily changes.

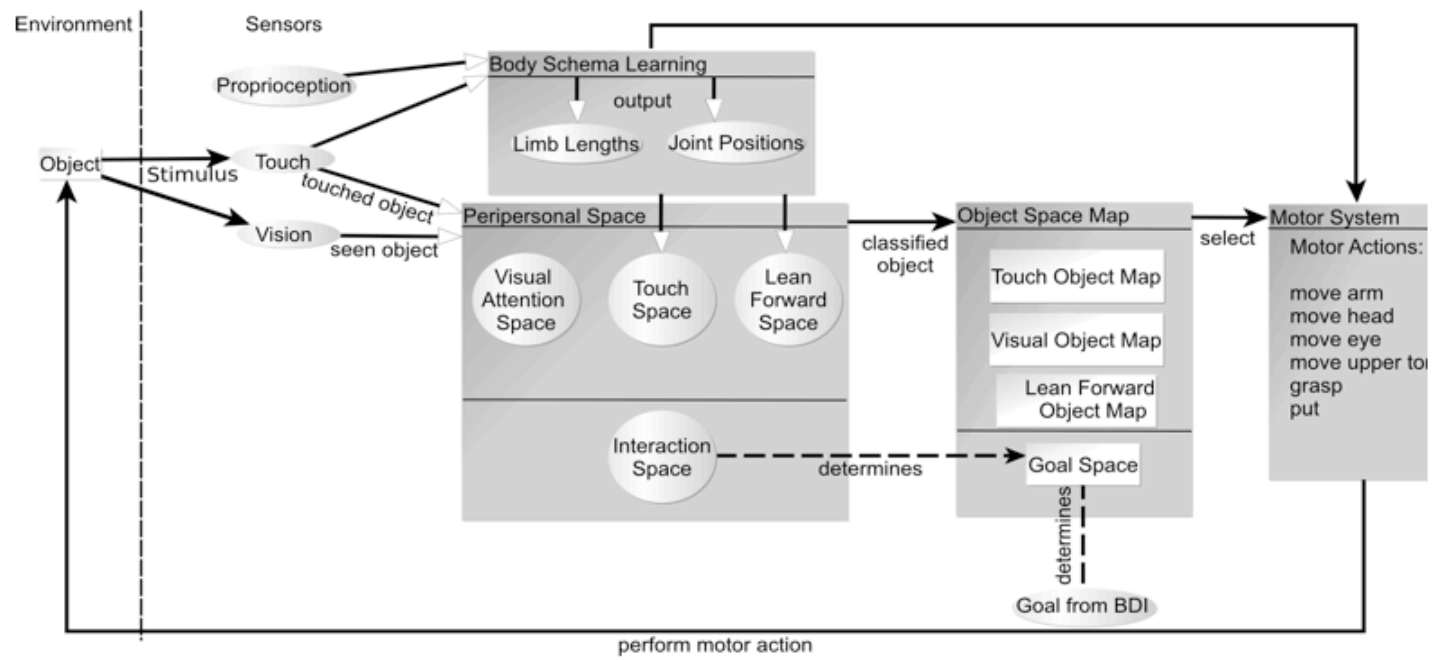

Figure 2: Technical Framework Overview. Information from body schema learning is utilized to build peripersonal subspaces. Objects perceived from different sensor modalities are classified into the subspaces and are maintained in object space maps. Objects outside the goal space induce a motor action, leading to a new sensor input.

\subsection{Peripersonal space}

In the technical framework one main aspect is the partition of the realm of peripersonal space into different subspaces according to findings from an interdisciplinary background. Extracted from the learned body schema they differ in spatial range, extent, and frames of reference. The core spaces are determined by their predominant sensor modality and comprise of a touch space, a lean-forward space, and a visual attention space. The subspaces are in line with the finding of multiple sensor modalities being involved in peripersonal space. In particular the lean-forward space 
allows for the gradual transition between peripersonal and extrapersonal space as described in Section 2.1. For a technical system, where sensor modalities do not necessarily cover the same spatial regions, the subspaces facilitate a more robust representation of peripersonal space. More details are described in Section 4.3.

\subsection{Object space maps}

Since an object can be perceived with different sensor modalities, it can be represented in different peripersonal subspaces. Each perceived object is maintained in object space maps, corresponding to the sensor modalities it was perceived from. The advantage is that the virtual human can keep track of whether objects are within its visual or touch space. Thus the virtual human can select its next movement, for example, forwardleaning or reaching for an object. As an additional spatial map we define a goal space within the peripersonal space. This space defines a region in peripersonal space, which the virtual human should direct its attention to, for example to objects related to a task on a table in front of the torso. The extent and location of the goal space can be determined through different factors, for instance, a new goal from the virtual human's Belief-Desire-Intention framework. The maintenance of the object space maps will be described in Section 4.3.

\subsection{Motor system}

Information about object positions from the object space maps is used to choose an appropriate motor action. For example, if an object has been touched, but not seen so far, the motor system will generate a head or eye movement in the direction of the touched object. By means of this, the visual attention space is shifted to cover the new object. If the object lies outside the goal space, a motor action is generated to grasp the object and put it into the current goal space.

\subsection{Interaction space}

If one or more articulated agents are entering the virtual human's peripersonal space, it assumes that they are also surrounded by a peripersonal space. The peripersonal spaces, in a first simple approach, are simulated as large as the peripersonal space of the virtual human. The overlapping spaces form the space reachable to all participants. In 
cooperative interaction this space is then marked as a new goal space. The virtual human would now centre its attention to the new space and would, for example, place objects into it, supporting the interaction. We describe this issue in Section 5.

\section{A computational model of peripersonal space for a humanoid}

In this section we present our computational model of peripersonal space for Max (Kopp et al., 2003), our virtual human. Multisensory abilities are a crucial factor in our framework, thus the demands we make on a virtual human's sensor system are described in Section 4.1. On the one hand sensor data are used to learn Max's kinematic structure using data from virtual touch and proprioception sensors, described in 4.2. On the other hand, since sensor modalities do not necessarily cover the same space, their combination accounts for establishing a comprehensive perception of Max's peripersonal space, described in Section 4.3. In the interaction scenarios we assume that peripersonal space interaction with objects usually involves a plane, lateral in front of a virtual human's body, for example, a table. In order to decrease the complexity of the model, we therefore focus on peripersonal space on a two-dimensional plane lateral, in front of Max's upper torso. The range of the spaces defined in Section 4.3 is thus projected on this two-dimensional plane.

\subsection{Sensor requirements for a virtual human}

In previous work (Nguyen and Wachsmuth, 2009), we developed and technically realized touch receptors for Max's whole virtual body. These receptors allow for differentiating between different qualities of tactile stimulation. Biological findings on the human tactile system were incorporated to build an artificial sense of touch for Max. The virtual skin consists of flat quadrangle geometries varying in size, each representing a single skin receptor. Altogether the virtual skin consists of more than 200 virtual skin receptors. Max's tactile system provides information on which body limb a virtual skin receptor is attached to, together with the position in the limb's frame of reference (FOR), allowing for determining where Max is being touched. In addition to the tactile system, the virtual agent's body has an underlying anthropomorphic kinematic skeleton which consists of 57 joints with 103 Degrees of Freedom altogether. Every time Max executes a movement, the joint angle information of the involved joints 
is output. Synchronously with the tactile information, the proprioceptive information can be observed.

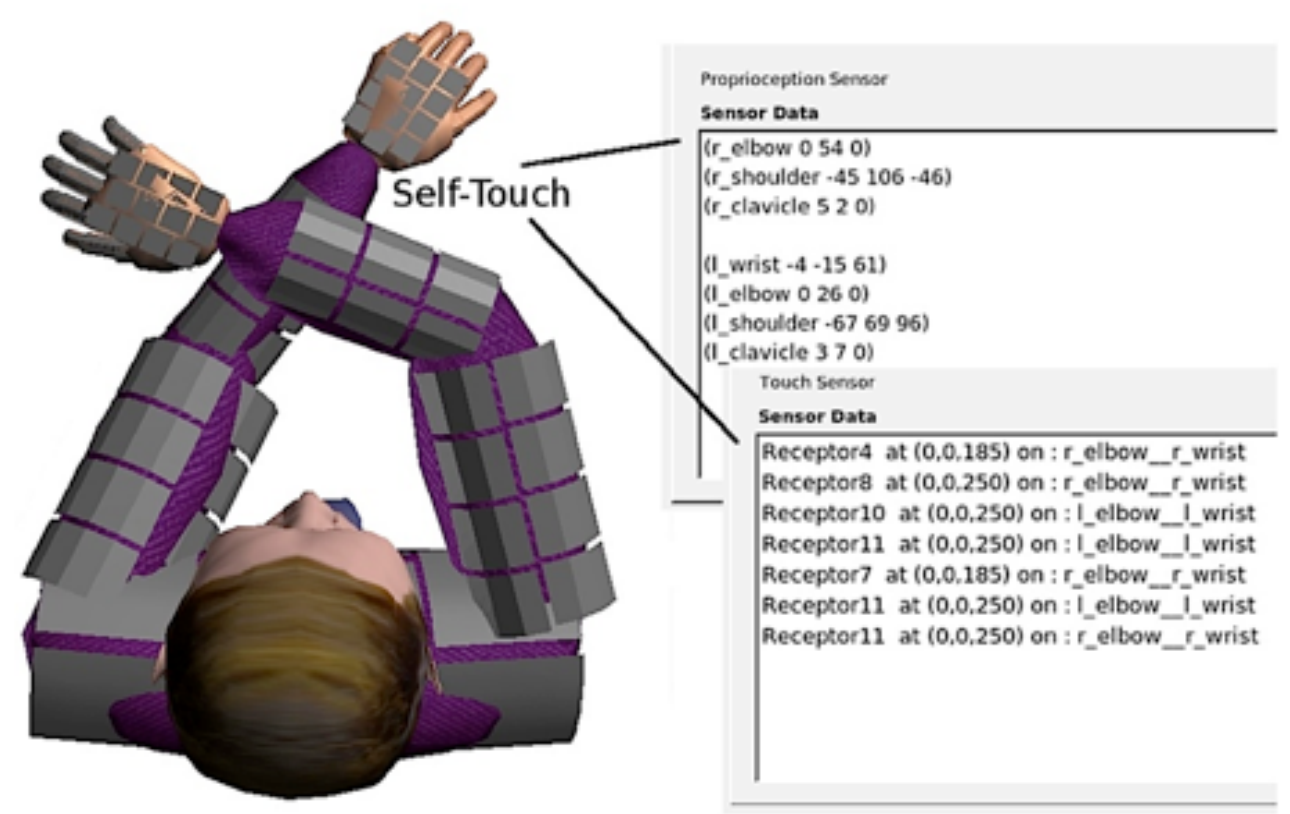

Figure 3: Tactile body schema learning: For each random posture, sensory consequences are output by the sensor systems. The touch sensor provides an ID of the receptor, the limb it is attached to, and the position in the frame of reference (FOR) of the corresponding limb. Angle data for the involved joints are output by the motor system, representing the proprioceptive information. (Figure taken from Nguyen and Wachsmuth (2009))

In this work, Max's virtual visual field of view corresponds to human stereoscopic vision (Previc, 1998), required for effective hand-eye coordination and thus is limited to an angle of $60^{\circ}$, lateral attached to his head. Head and torso movements are translated to the virtual visual field, changing its position. The angle of view is projected onto a two-dimensional plane when he is sitting or standing at a table. Objects perceived in his virtual view are represented in head-centred coordinates.

\subsection{Tactile body schema learning for a humanoid}

The model for learning the body structure takes input data given by touch sensors and joint angle data given by the proprioception sensors. In a first step, Max executes 
random motor actions resulting in random body postures. For each posture he perceives proprioceptive data from his joints and tactile stimuli when randomly touching positions on his body (see Figure 3). As described in our previous work (Nguyen and Wachsmuth, 2009) we consider the body schema as a tree of rigid transformations. In our case this kinematic tree is prescribed by the skeleton of the virtual human Max. In the initial kinematic tree, the number of joints linked in their respective order with the number of limbs are known, but the joint orientation and positions are unknown. In our model the touch receptors are attached to the limbs and their position is represented in the limb's FOR. In the kinematic tree representation, the touch receptors can therefore be represented as located along the edges.

In order to learn the real positions and orientations of the joints which also determine the limb lengths, we make use of the algorithm proposed by Hersch et al. (2008). It is a novel and general approach in online adapting joint orientations and positions in joint manipulator transformations. Our challenge in using this algorithm was to adapt it to a case different from the one to which it was originally applied. In our case we did not use visual and joint angle data, but instead replaced all visual information by tactile information in order to update all the transformations along the chains in the kinematic tree. Each time Max touches his positions on his body, the two involved skin receptors' positions are used as an input for the update algorithm.

Due to the fact that the approach takes knowledge from the body structure in advance and does not learn sensor-motor mapping, this learning method is in the strict sense a recalibration mechanism, which corresponds to the definition of body schema which adapts to changing body limbs. By means of this, the limb lengths of Max's articulated skeleton are learned and then used to determine Max's reaching distances (Nguyen and Wachsmuth, 2011) and thus, his peripersonal space boundaries can be determined as suggested in Section 2.1. This aspect is described in the next section.

\subsection{Structuring peripersonal space}

According to Previc's (1998) cognitive model, each type of realm egocentrically surrounding a human is associated with certain predominant behavioural actions, for instance, visuomotor object-manipulation is predominant in peripersonal space and locomotion in action extrapersonal space. More precisely, in his model he ascribes a set 
of sensory-perceptual and motor operations and a predominant FOR to each realm. In order to technically realize this idea, and focusing on peripersonal space only, we decomposed his definition of peripersonal space into three major sensor components, namely vision, touch, and proprioception. Each of them spans a realm with a specific extent, FOR and predominant motor actions.

In this section the technical framework outlined in Section 3 and in Figure 2 is specified in more detail. In Table 1 characteristics of the spanned three subspaces of peripersonal space are presented. The results from the learning algorithm described in the previous section determine the boundaries of the subspaces. In the next subsection we explain the content of the table and will describe in Section 4.3.2 how the subspaces influence spatial object maps. Finally, we show how the object maps together with motor actions, delineated in Section 4.3.4, satisfy a defined goal realm, which is specified in Section 4.3.3.

Table 1: Characteristics of sensory subspaces of a virtual human's peripersonal space

\begin{tabular}{llll}
\hline & Visual Attention Space & Touch Space & Lean-Forward Space \\
\hline Function & Visual search, visual control & Grasping, placing, manipulation & Grasping, placing \\
\hline 2D location, extent & & & \\
Vertical & & Lower field, Projection on frontal 2D plane & \\
Origin & Head & Shoulder, Trunk & Shoulder, Trunk \\
Lateral & Central $60^{\circ}$ & $360^{\circ}$ & Frontal $180^{\circ}$ \\
Radial & $0-2 \mathrm{~m}$ & Length: shoulder joint to hand palm & Length: hip to hand palm \\
\hline Frames of Reference & Head centered & Limb centered & Limb centered \\
\hline Motor Action & Head, eye movements & Arm movements & Upper Torso movements \\
\hline
\end{tabular}

\subsubsection{Subspaces in peripersonal space}

The subspaces we define within peripersonal space are deduced from Previc's (1998) work and are adopted to the technical conditions determined by Max's sensor system. The major sensor modalities assumed to be involved in peripersonal space are determining the three subspaces. Vision is mainly utilized in object search and visual manipulation control and determines a visual attention space. Touch is mainly utilized in object manipulation and grasping, determining a touch space. The function of proprioception is always utilized in peripersonal space, but plays a particular role in the placing and grasping of objects at the boundaries of peripersonal space when efforts have to be made by leaning forward; in our framework it therefore determines an additional lean-forward space. As mentioned previously, this subspace constitutes the gradual transition between peripersonal and extrapersonal space. 
The characteristics of peripersonal subspaces we define for our virtual human are listed in Table 1. Their technical counterparts are shown in Figure 2. Each subspace defined here is associated to a main function determining the predominant motor actions carried out in the specific subspace. As mentioned at the beginning of this section, the boundaries of the subspaces are projected on an assumed two-dimensional plane on a table in front of Max. Hence, the vertical extent of each subspace is projected on a lower radial $180^{\circ}$ two-dimensional plane. A schematic layout is depicted in Figure 4.

The visual attention space's origin lies in the centre of the head. Its lateral extent is projected to the touch and lean-forward spaces. Stimuli perceived in Max's $60^{\circ}$ field of view are represented in a head-centred frame of reference.

The touch space's boundary is limited to the lengths of the arm limbs which were learned from the body schema. It radiates from the trunk's centre with the maximal distance covering the range between the shoulder joints and the palms of the hands. Although tactile stimuli may also effect the back of the body, that is, touch space covers a space with a lateral extent of $360^{\circ}$ around the trunk's centre, in the following scenarios we only examine $180^{\circ}$ of the lateral, frontal space.

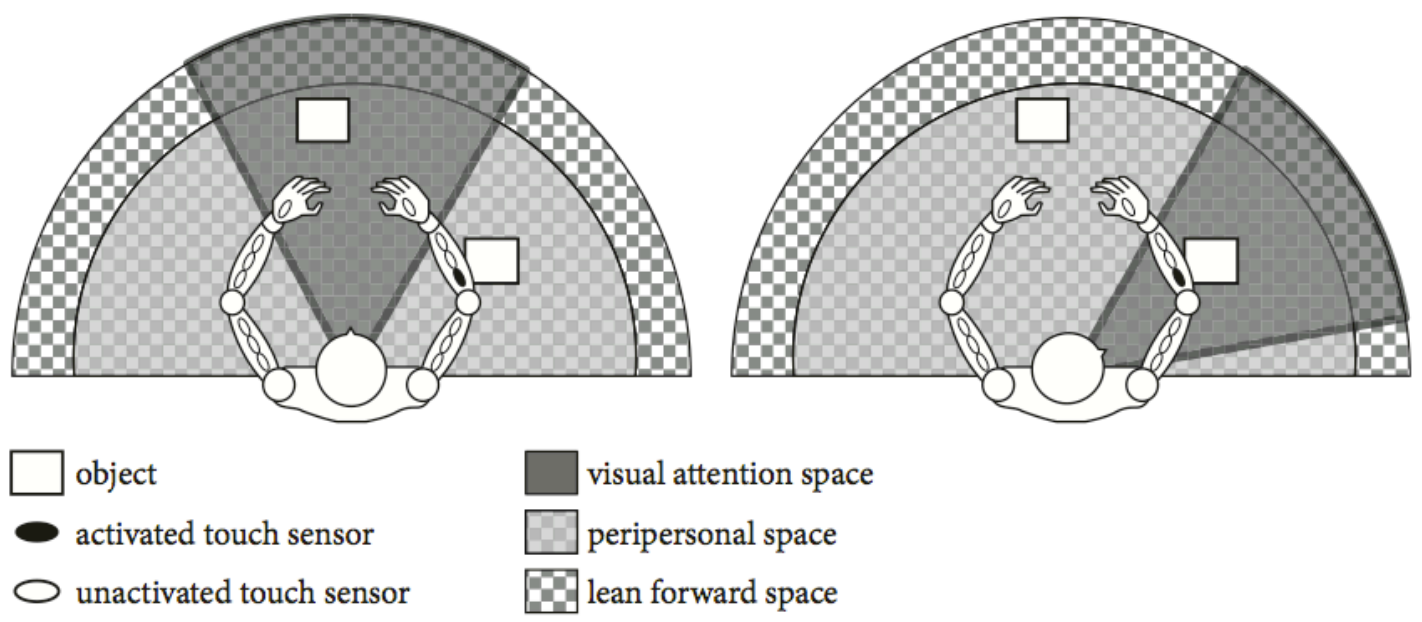

Figure 4: The virtual human directs its sensory attention to an object. Left: the virtual human perceives an object with the skin sensors beyond its visual attention space. The object is registered in the touch object map. Right: A motor action is selected and shifts the head and the visual attention space toward the touch-location. The object elicits a visual stimulus and is then registered to the visual object map. 
The lean-forward space's boundary is limited to the maximal reaching realm of the upper torso, when bending forward. From the learned body schema we compute the maximum range achieved with the arm limbs together with the spine joints which begin above the hip joint. This space thus extends touch space. Objects and stimuli perceived in both subspaces are represented in a limb-centred frame of reference. Compared to touch space, the function of object manipulation is not predominant in lean-forward space.

In addition to the mentioned spaces, other subspaces which potentially structure Max's peripersonal space can be established in our framework. As soon as other virtual or real human(s) enter Max's proximity, we assume that they are also surrounded by peripersonal spaces. The intersection of their overlapping peripersonal spaces are registered as an interaction space. Depending on the sensor modality from which an object was perceived, which subspaces the object is located in is evaluated. The classified object is then registered to the corresponding object space maps (see Figure 2).

\subsubsection{Object space maps}

Sensor information may differ in its reliability, for instance, the cause of a tactile stimulus might not be an object but be a person touching Max's arm to attract his attention. Thus, in order to keep track of the objects in Max's peripersonal space, the sensor modalities have to cover the objects, depending on a predefined sensor hierarchy. An example of objects being located in different peripersonal subspaces is shown in Figure 4. Although the literature reviewed by Avraamides et al. suggests the functional equivalence between perceptual-derived spatial representations in humans, the authors also state that the 'representational and computational underpinnings of equivalent performance' (Avraamides et al., this volume: 18) still needs to be clarified. In our framework we assume that the visual modality may provide more immediate information about objects - for example size and shape - than the touch modality, where acquisition of the same information requires more effort by grasping the object, exploring the surface, and building a mental image. Thus, visual search is preferred over tactile manipulation, and tactile manipulation is preferred over leaning forward. In the example shown in Figure 4, a virtual human like Max is accidentally touching, but not seeing a virtual object, since its visual attention space at that moment is not covering the object behind its arm. In our framework, the object would be listed in the touch-, but 
not in the visual- or lean-forward object map. Due to the mentioned hierarchy, a motor action would be triggered to sense the object with the visual modality. In this case a motor action is selected to turn the virtual human's head to the location where it touched the object, which leads the visual attention space to shift to the object location. If the stimulus was caused by an object, it is additionally registered to the visual map. In the current sensor hierarchy we consider factors like immediacy/effort and reliability in the acquisition of object information (e.g. position and size). According to such factors, further object properties such as audio (e.g. spatial sound is perceived from the direction of a newly placed object) or spatial language (e.g. the interaction partner verbally describes a newly placed object location) cues might also be integrated into the sensor hierarchy.

\subsubsection{Goal space}

In order to avoid collisions with objects when interacting, the virtual human may reorganize the object positions in its peripersonal space. For this purpose an additional spatial map, a goal space is defined, which describes his region of attention. In the example shown in Figure 4, we assume that the goal space is set to a default spatial region on the table, with an angle of $60^{\circ}$ central in front of the virtual human, so that objects are easy to see, reach, and touch, and the virtual human's motions are less prone to hindrances. All sensor modalities have a preference to cover the goal space as long as no external spatial interferences or constraints are given. Each time an object is perceived, the goal space map is compared to the object space maps. If differences between the maps are found, a motor action is selected to bring the virtual objects into Max's current goal space. In the schematic layout on the left in Figure 4 the default goal space is the space where visual attention and touch space overlap. Due to the preferences defined for the sensor modalities, the virtual human would turn its head to the location where the touch stimulus occurred. In a next step, due to the goal space definition, described in detail in Section 4.4, another motor action is triggered to grasp and put the object into the goal space.

\subsubsection{Motor actions}

As outlined in the previous example, motor actions are selected depending on the subspaces. Another factor in the selection of the appropriate motor action is the 
superposed potential fields, which is the topic of the next section. In touch space arm movements are predominant motor actions for fulfilling the functions of grasping, placing, and manipulation. In lean-forward space, arm movements are combined with upper torso movements, like leaning forward, in order to grasp for or place an object. Object manipulation is not predominant in this space, since objects are more likely to be brought to touch space. Visual attention space relies on motor actions like eye movements to control the gaze and head movements to shift the entire space. Furthermore, the replacement of objects relies on the information of the potential fields defined by the goal spaces. The information from the body schema is used to translate object positions from one frame of reference to another, since the subspaces code objects in different coordinate systems.

\subsection{Selecting actions in peripersonal space with potential fields}

In order to trigger appropriate motor actions with regard to objects at each location in peripersonal space, we used the method of artificial potential fields. This method is very common in obstacle avoidance and motion and path planning for artificial agents (Khatib, 1986). An artificial potential field is an array of vectors, which defines a spatial region in which each location of the field is exposed to a force vector, describing the direction and the strength of the radiating force. For example an object's direction and the velocity of a motion can be controlled depending on the length and the direction of the force vector. Multiple potential fields can be defined for the same spatial region. By adding the fields together, a new field with attenuated or amplified forces is built.

Goal space and Max's peripersonal space are modelled as artificial potential fields. The peripersonal space is described as a repulsive field $F_{p e r i}$, defined by Equation 1 with tangential directions covering a semicircle, defined by Equation 2. The field is visualized in Figure 5, left. A vector between the centre of peripersonal space and any location in space is denoted by position vector $\mathbf{p}$. We calculate the force vector $\mathbf{v}_{\text {peri }}(\mathbf{p})$, that is currently affecting $\mathbf{p}$, using Equation 3. The parameter $\xi$ denotes a positive scalar which influences the length of the resulting force vector. The force vectors $\mathbf{v}_{\text {peri }}(\mathbf{p})$ point to the frontal, sagittal mid-line, described by vector $r_{\text {perimid }}$. The field covers all ps within an angle of $90^{\circ}$ to both sides of this mid-line. The regions beyond the radius $r_{\text {peri }}$ of peripersonal space are not affected by the potential field. 
Therefore any $\|\mathbf{p}\|$ that is greater than $r_{\text {peri }}$ results in a zero force vector. The default goal space is modelled as a selective attractive field $F_{\text {goal }}$ defined by Equation 3 . The field covers the angle $\Theta_{\text {goal }}$ with an angle bisector denoted by $r_{\text {goalmid }}$, and force vectors pointing away from the centre in (see Equation 4). The default goal space has an angle of $\Theta_{\text {goal }}=60^{\circ}$, and is visualized in Figure 5, middle. The sum of the two fields are shown in Figure 5, right.

Each time Max perceives an object, the current force vector $\mathbf{v}_{r e s}$ impacting on the object is calculated using Equation 5. Objects outside the goal space, that have to be relocated, would be affected by force vectors, describing a path which leads in the direction of the inside of the goal space. With decreasing distance to the centre, the strength of the potential field disappears, ending the path. Max is not exactly following the path, but uses the force vectors as a trigger to select a grasping motion. The end position of the path is used as a target position for a placing motion. Objects located within goal space are represented with repulsive potential fields, which prevents new objects being placed at their location. This example shows that potential fields are a suitable method to associate each point in peripersonal space to a specific behaviour, in this case motor actions. By superposing several potential fields, behaviours can be combined, allowing for more sophisticated actions. It is important to note that the potential fields facilitate the selection of an appropriate action with regard to the space it is executed in. The question of whether and when the action is executed is determined by the actual situation and interaction context.
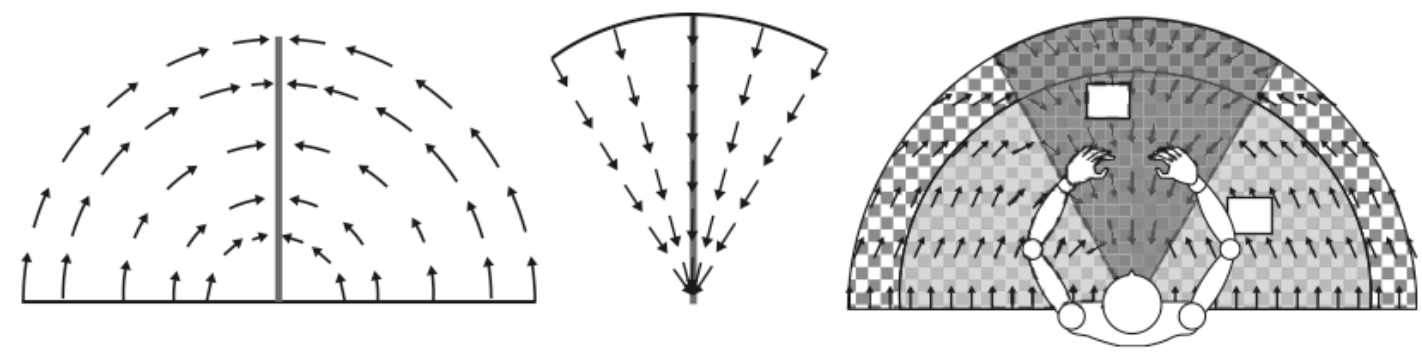

Figure 5: Peripersonal space modelled as tangential potential field with $r_{\text {perimid }}$ depicted as a grey line. Middle: Default goal space modeled as selective attraction field with an angle $\Theta_{\text {goal }}$ of $60^{\circ}$ and $r_{\text {goalmid }}$ depicted as a grey line. Right: Addition of the two fields shows the resulting peripersonal space field. 
Goal spaces in general can be determined by a new goal, raised by the BeliefDesire-Intention system or by a new established subspace of the peripersonal space. In particular a new established interaction space as described in Section 4.3.1 holds interesting potential field combinations and associated motor actions that we describe in Section 5.2.

(1)

$$
\mathbf{F}_{\text {peri }}(\mathbf{p})= \begin{cases}\xi\left(\frac{1}{\|\mathbf{p}\|}-\frac{1}{r_{p e r i}}\right) \frac{\mathbf{p}}{\|\mathbf{p}\|^{3}} & \|\mathbf{p}\| \leq r_{\text {peri }} \\ 0 & \|\mathbf{p}\|>r_{\text {peri }}\end{cases}
$$

(2)

$$
\mathbf{v}_{\text {peri }}(\mathbf{p})= \begin{cases}-\left(\frac{\pi}{2}\right) * \mathbf{F}_{\text {peri }}(\mathbf{p}) & \forall \mathbf{p} \mid \measuredangle\left(\mathbf{r}_{\text {perimid }}, \mathbf{p}\right) \leq-\left(\frac{\pi}{2}\right), \\ \left(\frac{\pi}{2}\right) * \mathbf{F}_{\text {peri }}(\mathbf{p}) & \forall \mathbf{p} \mid \measuredangle\left(\mathbf{r}_{\text {perimid }}, \mathbf{p}\right) \leq\left(\frac{\pi}{2}\right), \\ 0 & \text { else }\end{cases}
$$

(3)

$$
\mathbf{F}_{\text {goal }}(\mathbf{p})=-\xi \frac{\mathbf{p}}{\|\mathbf{p}\|}
$$

(4)

$$
\mathbf{v}_{\text {goal }}(\mathbf{p})= \begin{cases}\mathbf{F}_{\text {goal }}(\mathbf{p}) & \forall \mathbf{p} \mid \measuredangle\left(\mathbf{r}_{\text {goalmid }}, \mathbf{p}\right) \leq\left(\frac{\Theta_{\text {goal }}}{2}\right), \\ 0 & \text { else }\end{cases}
$$

$(5)$

$$
\mathbf{v}_{\text {res }}(\mathbf{p})=\mathbf{v}_{\text {peri }}(\mathbf{p})+\mathbf{v}_{\text {goal }}(\mathbf{p})
$$

\section{A computational model of interaction space for a humanoid}

Until now we have modelled the individual peripersonal space for a virtual human with potential fields to control its actions. We will now propose how to computationally model the space between a virtual human and its interaction partners. As mentioned previously, we base our work on Kendon's F-formation system.

\subsection{Extending the F-formation system}

With our model we aim to supplement the F-formation system by adding the aspect of a measurable shared space, suitable for computational applications. In Figure 6 we show how we modelled the space between interactants. Compared to Figure 1, Kendon's o- 
space is now defined as the intersection of the interactants' overlapping peripersonal spaces (Figure 6, striped regions). We define this space as their interaction space. Since our definition is the intersection of all interactants' reaching realm, it conforms to Kendon's definition of the interactive space as that which is equally and exclusively reachable to all interactants, and in which they cooperate. In order for a virtual human to sustain an F-formation arrangement, once it is established, we incorporate interaction space into our described framework.

When Max perceives an interactant within an F-formation, he projects his own peripersonal space to the partner, in order to build a representation of the partner's reaching space. This process is similar to a mechanism in human cognition which is usually referred to as spatial perspective taking. By means of spatial perspective taking, Max is able to build a hypothesis on how far the partner can reach with her hands or by additionally leaning forward with the upper part of her body. Together with the representation of the objects, Max builds a hypothesis on which objects are or are not reachable for the partner. The fact that Max is simulating the partner's perspective by using his own body structure is commonly known as embodied simulation (Gallese, 2005) and is a hypothesis of how humans understand others. Gallese views the mechanism of embodied simulation as one basis for social cognition. Studies by Kessler and Thomson (2010) support the position that spatial perspective taking is an embodied cognitive process which might still be rooted in embodied representations, which supports our approach. However, at the current stage, in the framework we project Max's peripersonal boundaries to another partner's body structure manually. Further steps are discussed in Section 6.
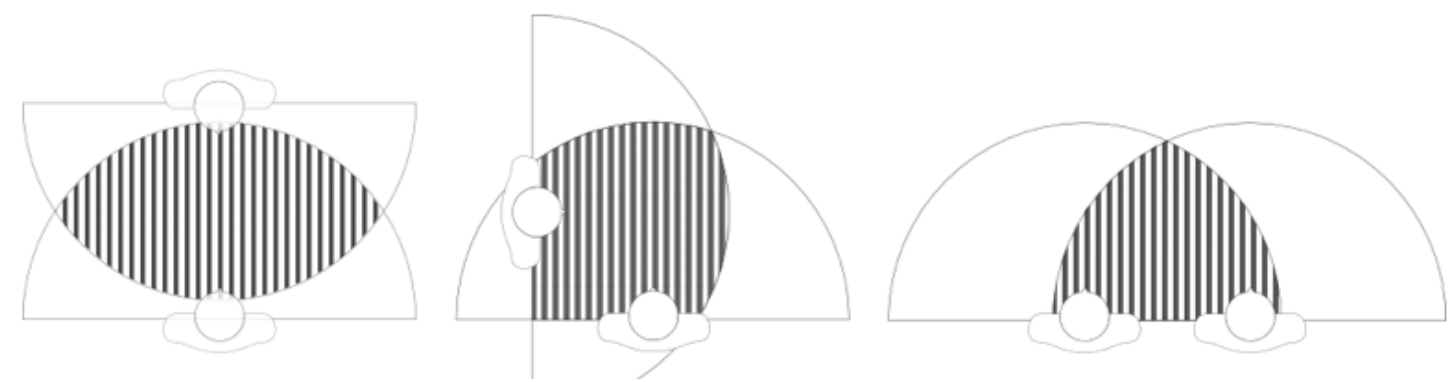

Figure 6: Kendon's o-spaces modeled as interaction spaces (striped regions). Interaction spaces are established by the intersection of the interactants' overlapping peripersonal spaces. 

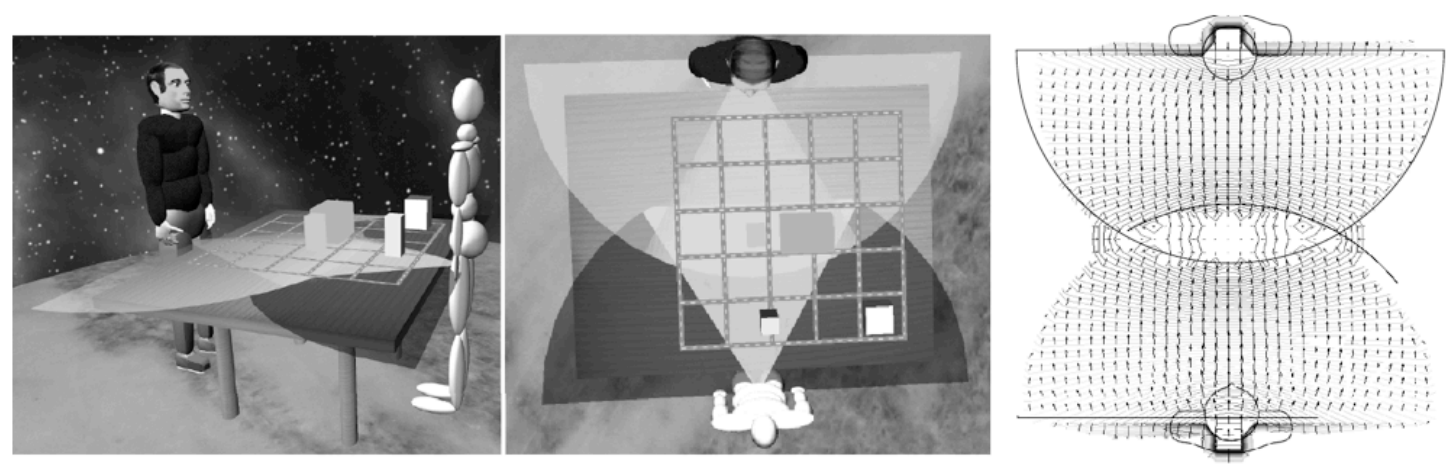

Figure 7: Left: Max (left) and an articulated humanoid (right) interacting in a virtual environment with visualized peripersonal subspaces. Middle: Bird-view perspective in the vis-à-vis arrangement with interaction space between the interactants. Right: The resulting potential field as a superposition of interactants' selective repulsive fields and one attractive potential field within interaction space.

\subsection{Selecting and modulating actions in interaction space with potential fields}

Figure 7 (left) shows a vis-à-vis F-formation between Max and another articulated humanoid in a virtual reality scenario. In this scenario both partners are standing at a table and cooperate in an object manipulation task where they have to cooperatively build a tower with toy blocks. The tower is to be located in the partners' interaction space. In order to build the tower, the partners have to put one tower block upon the other. A newly placed tower block has to be smaller than the previously placed one. The tower blocks are randomly placed at free locations within the partners' peripersonal spaces. In this scenario, Max helps his partner, for example, by placing into the interaction space a tower block which is not within reach for but is needed by his partner.

As soon as Max's and his partner's peripersonal subspaces overlap (see Figure 7, middle) an interaction space is established, which is defined as the new goal space. Therefore Max directs his sensory attention to this space. Max's and the partner's peripersonal spaces are modelled as selective repulsive potential fields, as shown in Equation 1. Their interaction space is modelled as an attractive potential field $F_{\text {inter }}$, as described in Equation 3, with its centre being the centre of a circle, which approximates interaction space. The range of the $F_{\text {inter }}$ covers all interactants' potential fields. Thus, each force vector within their peripersonal spaces is distracted in the direction of the 
interaction space, as depicted in Figure 7, right. Within interaction space, the field strength disappears so that objects are placed within the space. If Max is currently performing a motor action to place a tower block into interaction space, the partner could in parallel lean forward to place another tower block. This would immediately result in a larger interaction space, which leads Max's current placing motion to end before the initially planned one. Thus, interferences between Max's and his partners actions are avoided without Max having to interrupt his entire action.

\subsubsection{Modelling cooperation and competition in F-formations}

In the scenario described so far, Max acts in a cooperative way as soon as an Fformation yielding an interaction space is established. The fact that Max's peripersonal space is modelled as a repulsive potential field, can be interpreted as his potential to share tower blocks with his partner, that is, to put tower blocks into interaction space, where they are accessible to the partner. However, Max's cooperative behaviour can be modulated, or even be inverted to competitive behaviour. The following parameters can be changed to control Max's behaviour.

The radius $r_{p e r i}$ of the potential field in Equation 1 is related to the end point of the chosen motor action. By decreasing or increasing the radius of the field, the distance of a chosen motor action can be modulated. This may trigger motor actions associated with more effort, for example bending of the whole torso to lean forward. The more effort Max makes to help his partner - that is to place objects nearer to the partner - the more his action could be interpreted as helpful. Decreasing the parameter $\xi$ in Equation 1 leads to a less repulsive field, whereas increasing $\xi$ leads to a more repulsive field which again decreases or increases the lengths of the resulting force vectors. In our approach, force vector lengths control the velocity of a chosen motor action. A chosen motor action affected by a strong repulsive field would be executed in a fast motion, whereas a less repulsive field would induce a slower motion. In the example of Max being helpful by placing objects nearer to the partner, a slow motion could be interpreted as Max being more reluctant, whereas a faster motion could be interpreted as Max being more cooperative. Finally, changing the repulsive field into an attraction field may reveal Max's competitive behaviour by taking all the objects from the interaction space to his peripersonal space, where only he can access them. 
With our presented approach we aim to make two contributions. The first contribution is a computational model of peripersonal space to be employed by an artificial agent. The model allows for a multi-modal and comprehensive perception of the space immediately surrounding the agent's body and for naturally structuring the agent's behaviour. The second contribution is a model of interaction space suitable for coordinating an agent's actions performed in shared space in the pursuit of cooperative tasks. While the approach could also serve for a physical robot, our methods were developed for a virtual human.

In a first step we realized the individual body space of a virtual human in terms of a multi-sensory representation, involving touch, vision, and proprioception. This concept, commonly known as peripersonal space, takes its information from the body structure, known as body schema. Changes in body schema also affect peripersonal space, which we realized by a recalibration algorithm. In a second step we divided peripersonal space into subspaces corresponding to each sensor modality. In a third step we modelled the behaviour within peripersonal space and interaction space. The method of potential fields proves to be applicable not only for modelling the peripersonal space of a virtual human, but also for the space it shares with others, that is, the interaction space. This aspect corresponds to work by Krueger (2011) who raises the issue of connecting individual and interpersonal space in order to study social cognition. Finally, we showed how our model of interaction space for virtual humans supports their cooperative behaviour in shared space and also implies a broader range of social behaviour. With this work we demonstrate how virtual partners can show adequate spatial behaviour even in limited near-space interaction to facilitate the accomplishment of cooperative tasks. In the future, Max's spatial behaviour has to be evaluated in cases where the partner's actions are very quick. Max's performance should then also keep up with the course of the interaction. Another issue is to evaluate Max's perception when his peripersonal space is crowded with objects. Max should then still be able to form an adequate representation of the objects within peripersonal space.

Further technical work is conceivable in order to enable Max to infer the spatial perspectives of the partners and to anticipate their actions. Work on perceptual spatial perspective taking, together with action recognition between two robots, has been 
presented by Johnson and Demiris (2005). Work on action anticipation was presented by Gray et al. (2005) in which the robot Leonardo anticipates a human's task plans in a cooperative interaction task. However, their robots are not humanoid and the two works need to be modified and combined to be applicable to spatial interaction scenarios as described in this chapter. Another aspect to be improved was mentioned previously: in the interaction scenario between Max and another humanoid partner, Max's peripersonal space boundaries are manually projected to another partner's body structure. In a next step Max should automatically recognize the partner's bodily structure and then simulate the partner's peripersonal space. Max could also adapt to the individual bodily differences by inferring and ascribing, for instance, a smaller reaching space to people with shorter arms. By simulating the peripersonal spaces of the partners, Max may, for instance, infer whether objects are obstructed or not reachable from the partners' perspectives and hence may better help them.

One final aspect we want to address is the multi-modal representation of peripersonal space. In Section 4.2.3 we mentioned the possibility of incorporating into the framework further modalities, such as auditory and lingustic input, in order to build a more comprehensive spatial representation of Max's surroundings. However, linguistically derived spatial representations in technical systems have to be carefully modelled in terms of computational performance and its contribution beyond the perceptual derived spatial cues. Such factors need to be analysed to assure consistent sensory attention and spatial behaviour of embodied agents like Max. More discussion about the relation between perceptual and linguistic space representation is presented by Avraamides et al. (this volume). We agree with Avraamides and colleagues that a more comprehensive understanding of how different modalities establish space representations in humans improves the spatial behaviour of technical systems. We consider this viewpoint as important in enabling humanoids like Max to support humans in spatial interaction tasks.

\section{Acknowledgements}

This work has been kindly supported by the Collaborative Research Center 673 Alignment in Communication, funded by the German Research Foundation (DFG). We thank Kim-Anh Tran for assisting in the research project and this publication. 
This chapter has appeared in T. Tenbrink, J. M. Wiener, and C. Claramunt (Eds.), Representing Space in Cognition (Chapter 8). Oxford University Press, 2013.

\section{References}

Biocca, F., Tang, A., Owen, C., Mou, W., and Fan, X. (2005). Mobile infospaces: personal and egocentric space as psychological frames for information organization in augmented reality environments. In D. D. Schmorrow (ed.), Foundations of Augmented Cognition (Proceedings of HCI International 2005, Vol. 11, pp. 154-163). Mahwah, NJ: Lawrence Erlbaum Associates.

Caggiano, V., Fogassi, L., Rizzolatti, G., Thier, P., and Casile, A. (2009). Mirror neurons differentially encode the peripersonal and extrapersonal space of monkeys. Science, 324, 403-406.

Fuke, S., Ogino, M., and Asada, M. (2007). Body image constructed from motor and tactile images with visual information. International Journal of Humanoid Robotics, 4(2), 347-364.

Fuke, S., Ogino, M., and Asada, M. (2009). VIP neuron model: head-centered crossmodal representation of the peri-personal space around the face. In Proceedings of the 7th IEEE International Conference on Developmental Learning (pp. 145$150)$.

Gallagher, S. (2005). How the Body Shapes the Mind. Oxford: Clarendon Press.

Gallese, V. (2005). Embodied simulation: From neurons to phenomenal experience. Phenomenology and the Cognitive Sciences, 4(1), 23-48.

Goerick, C., Wersing, H., Mikhailova, I., and Dunn, M. (2005). Peripersonal space and object recognition for humanoids. In Proceedings of the IEEE/RSJ International Conference on Humanoid Robots (Humanoids 2005), Tsukuba, Japan (pp. 387392). Washington, DC: IEEE Press.

Gray, J., Breazeal, C., Berlin, M., Brooks, A., and Lieberman, J. (2005). Action parsing and goal inference using self as simulator. In Proceedings of the Fourteenth IEEE Workshop on Robot and Human Interaction (pp. 202-209).

Hall, Edward T. (1966). The Hidden Dimension. New York: Anchor Books. 
Hersch, M., Sauser, E., and Billard, A. (2008). Online learning of the body schema. International Journal of Humanoid Robotics, 5(2), 161-181.

Holmes, N. and Spence, C. (2004). The body schema and multisensory representation(s) of peripersonal space. Cognitive Processing, 5(2), 94-105.

Huang, Z., Eliëns, A., and Visser, C. T. (2003). Is it within my reach?-an agents perspective. In T. Rist, R. S. Aylett, D. Ballin, J. Rickel, (eds), Proceedings of the 4th International Workshop Intelligent Virtual Agents (pp. 150-158). Berlin: Springer (LNAI 2792).

Johnson, M. and Demiris, Y. (2005). Perceptual perspective taking and action recognition. International Journal of Advanced Robotic Systems, 2(4), 301-308.

Kendon, A. (1990). Conducting Interaction. London: Cambridge University Press.

Kessler, K. and Thomson, L. A. (2010). The embodied nature of spatial perspective taking: embodied transformation versus sensorimotor interference. Cognition, 114(1), 72-88.

Khatib, O. (1986). Real-time obstacle avoidance for manipulators and mobile robots. International Journal of Robotics Research, 5(1), 90-98.

Kopp, S., Jung, B., Leßmann N., and Wachsmuth, I. (2003). Max-A multimodal assistant in virtual reality construction. KI-Künstliche Intelligenz, 4(3), 11-17.

Krueger, J. (2011). Extended cognition and the space of social interaction. Consciousness and Cognition, 20(3), 643-657.

Lloyd, D. M. (2009). The space between us: A neurophilosophical framework for the investigation of human interpersonal space. Neuroscience and Biobehavioral Reviews, 33(3), 297-304.

Longo, M. R. and Lourenco, S. F. (2006). On the nature of near space: effects of tool use and the transition to far space. Neuropsychologia, 44, 977-981.

Longo, M. R. and Lourenco, S. F. (2007). Space perception and body morphology: Extent of near space scales with arm length. Experimental Brain Research, 177, pp. 285-290.

Maravita, A. and Iriki, A. (2004). Tools for the body (schema). Trends in Cognitive Science, 8, 79-86.

Nabeshima, C., Kuniyoshi, Y., and Lungarella, M. (2006). Adaptive body schema for robotic tool-use. Advanced Robotics, 20(10), 1105-1126. 
Nguyen, N. and Wachsmuth, I. (2009). Modeling peripersonal action space for virtual humans using touch and proprioception. In Z. Ruttkay, M. Kipp, A. Nijholt, and H. H. Vilhjálmsson (eds), Proceedings of the 9th Conference on Intelligent Virtual Agents (pp. 63-75). Berlin: Springer (LNAI 5773).

Nguyen, N. and Wachsmuth, I. (2011). From body space to interaction spacemodeling spatial cooperation for virtual humans. In Tumer, Yolum, Sonenberg, and Stone (eds), Proceedings of the 10th International Conference on Autonomous Agents and Multiagent Systems (AAMAS 2011), (pp. 1047-1054) Taipei, Taiwan.

Pedica, C. and Vilhjálmsson, H. H. (2009). Spontaneous avatar behavior for human territoriality. In Z. Ruttkay, M. Kipp, A. Nijholt, and H. H. Vilhjálmsson (eds), Proceedings of the 9th Conference on Intelligent Virtual Agents (pp. 344-357). Berlin: Springer (LNAI 5773).

Previc, F. H. (1998). The neuropsychology of 3-D space. Psychological Bulletin, 124(2), 123-164.

Rickel, J. and Johnson, W. L. (2000). Task-oriented collaboration with embodied agents in virtual worlds. In J. Cassell, J. Sullivan, S. Prevost, and E. Churchill (eds), Embodied Conversational Agents (pp. 95-122). Cambridge, MA: MIT Press.

Sisbot, E. A., Marin, L. F., Alami, R., and Simeon, T. (2006). A mobile robot that performs human acceptable motion. In Proceedings of the IEEE/RSJ International Conference on Intelligent Robots and Systems (pp. 1811-1816).

Vallar, G. and Maravita, A. (2009). Personal and extrapersonal spatial perception. In G. G. Berntson and J. T. Cacioppo (eds), Handbook of Neuroscience for the Behavioral Sciences (pp. 322-336). Hoboken, NJ: John Wiley \& Sons, Inc.

Yamaoka, F., Kanda, T., Ishiguro, H., and Hagita, N. (2008). How close?: Model of proximity control for information-presenting robots. In Proceedings of the $3 \mathrm{rd}$ ACM/IEEE International Conference on Human-Robot Interaction (pp. 140144). New York: ACM.

Zhao, L., Liu, Y., and Badler, N. I. (2005). Applying empirical data on upper torso movement to real-time collision-free reach tasks. In Proceedings of the 2005 SAE Digital Human Modeling for Design and Engineering Conference and Exhibition (pp. 2885-2890). Berlin: Springer. 